\title{
CARACTERÍSTICAS CLÍNICAS Y EPIDEMIOLÓGICAS DE NIÑOS CON INFECCIÓN POR SARS-COV-2 INTERNADOS EN UN HOSPITAL PERUANO
}

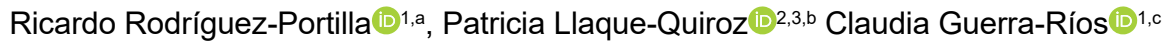 \\ Liliana Paola Cieza-Yamunaqué (1)1,a, Edgar Juan Coila-Paricahua ${ }^{11, a}$, \\ Pedro Michael Baique-Sánchez (iD) 1,a, Isabel Pinedo-Torres (iD) 4,d \\ 1 Hospital Nacional Edgardo Rebagliati Martins, Lima, Perú. \\ 2 Instituto Nacional de Salud del Niño San Borja, Lima, Perú. \\ 3 Universidad Peruana de Ciencias Aplicadas, Lima, Perú. \\ ${ }^{4}$ Facultad de Ciencias de la Salud, Universidad Cientifica del Sur, Lima, Perú. \\ a Médico/a pediatra intensivista; ${ }^{\mathrm{b}}$ médica pediatra neumóloga, magíster en ciencias de investigación epidemiológica; \\ ${ }^{c}$ médica pediatra; ${ }^{\mathrm{d}}$ médica endocrinóloga.
}

\section{RESUMEN}

Con el objetivo de conocer las características clínicas y epidemiológicas de niños con infección por SARS-CoV-2 internados en un hospital peruano de referencia nacional realizamos un estudio observacional, retrospectivo y descriptivo e incluimos pacientes de un mes a catorce años hospitalizados entre marzo a agosto del 2020. Se ingresaron 125 pacientes con infección por SARS-CoV-2, el 18,4\% $(\mathrm{n}=23)$ presentaron enfermedad crítica y $16,8 \%(\mathrm{n}=21)$ síndrome inflamatorio multisistémico (SIM). En los pacientes con SIM fue más frecuente la ausencia de comorbilidades y el antecedente de contacto epidemiológico. Tanto el grupo en estado crítico como del grupo con SIM, en comparación con los que no tuvieron estas condiciones, presentaron menores recuentos de linfocitos y plaquetas, y mayores valores de proteína C reactiva, ferritina y dímero D. Seis (4,8\%) niños de los 125 fallecieron, 3 (13\%) del grupo en estado crítico y ninguno del grupo con SIM.

Palabras clave: SARS-CoV-2; Síndrome Mucocutáneo Linfonodular; Enfermedad Crítica; Epidemiología; Signos y Síntomas; Niños (fuente: DeCS BIREME).

Citar como: Rodríguez-Portilla R, Llaque-Quiroz P, Guerra-Ríos C, Cieza-Yamunaqué LP, CoilaParicahua EJ, Baique-Sánchez $\mathrm{PM}$, et al. Características clínicas y epidemiológicas de niños con infección por SARS-CoV-2 internados en un hospital peruano. Rev Peru Med Exp Salud Publica. 2020;38(2):2616. doi: https://doi.org/10.17843/ rpmesp.2021.382.6719.

\section{Correspondencia:}

Ricardo Rodriguez Portilla, ricardo.rodriguez@upch.pe

Recibido: 09/11/2020 Aprobado: 12/03/2021 En Línea: 19/05/2021

\section{CLINICAL AND EPIDEMIOLOGICAL CHARACTERISTICS OF CHILDREN WITH SARS-COV-2 INFECTION ADMITTED TO A PERUVIAN HOSPITAL}

\begin{abstract}
We carried out an observational, retrospective and descriptive study in order to identify the clinical and epidemiological characteristics of children with SARS-CoV-2 infection admitted to a Peruvian national referral hospital. We included patients from one month old to fourteen years old hospitalized between March and August 2020. A total of 125 patients with SARS-CoV-2 infection were admitted, 18.4\% $(\mathrm{n}=23)$ had critical illness and $16.8 \%(\mathrm{n}=21)$ had multisystem inflammatory syndrome (MIS-C). The absence of comorbidities and previous history of epidemiological contact were more frequent in patients with MIS-C. Patients in critical condition and patients with MIS-C had lower lymphocyte and platelet counts, and higher C-reactive protein, ferritin and D-dimer values than patients who did not have said conditions. Six (4.8\%) out of 125 children died, as well as $3(13 \%)$ children from the group of patients in critical condition. None of the children with MIS-C died.
\end{abstract}

Keywords: SARS-CoV-2; Mucocutaneous Lymph Node Syndrome; Critical Illness; Epidemiology; Signs and Symptoms; Children (source: MeSH NLM). 


\section{INTRODUCCIÓN}

La enfermedad por coronavirus (COVID-19) fue declarada pandemia por la Organización Mundial de la Salud el 11 de marzo del $202{ }^{(1)}$. A nivel mundial se reportan más de 116 millones de casos con 2,6 millones de fallecimientos ${ }^{(2)}$. La COVID-19 afecta más a la población adulta que a la pediátrica

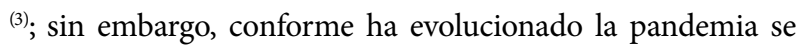
reportan series de casos graves y hasta fatales en niños ${ }^{(4-6)}$. Además, en este grupo, la presentación clínica de la infección por SARS-CoV-2 puede asociarse a un síndrome inflamatorio multisistémico (SIM) con compromiso multiorgánico de repercusiones graves ${ }^{(7-10)}$.

Dada la gravedad de esta enfermedad y su comportamiento aún no del todo conocido en la población pediátrica, se justifican los esfuerzos por describir el perfil clínico y epidemiológico de los niños hospitalizados con infección por SARS-CoV-2, prestando atención a los que desarrollaron enfermedad crítica y SIM. Conocer las características de esta población con mayores necesidades de salud, ayudaría a mejorar la planificación de su atención en nuestra realidad. Por ello, el objetivo de esta investigación fue describir las características clínicas y epidemiológicas de niños con infección por SARS-CoV-2 internados en un hospital peruano de referencia nacional.

\section{EL ESTUDIO}

\section{Diseño y población de estudio}

Se realizó un estudio observacional retrospectivo y descriptivo. La población diana son los pacientes pediátricos que se atienden en el Hospital Nacional Edgardo Rebagliati Martins en Lima, Perú. Este hospital es un centro de referencia nacional que brinda atención a 2 millones de peruanos con seguro social y cuenta con más de 1600 camas ${ }^{(11)}$, el área de pediatría tiene 100 camas en hospitalización y 9 en la unidad de cuidados intensivos pediátricos (UCIP).

La muestra estuvo conformada por pacientes de 1 mes hasta 13 años con 11 meses y 29 días internados por cualquier causa entre el 16 de marzo (inicio de la emergencia sanitaria nacional en Perú) y el 31 de agosto del 2020, a quienes se les confirmó el diagnóstico de infección por SARS-CoV-2. Se excluyeron a los pacientes que permanecieron, desde el ingreso hasta el alta, solo en el área de Emergencia Pediátrica y no accedieron a una cama en hospitalización general o UCIP sea por falta de disponibilidad o por la menor severidad de su enfermedad.

Se siguieron las recomendaciones del Ministerio de Salud del Perú para definir como caso de infección por SARS-CoV-2 a todo paciente con una prueba serológica o molecular positiva $^{(12)}$. La presencia de SIM se agregó al diagnóstico si el paciente presentó fiebre, proteína $\mathrm{C}$ reactiva (PCR) elevada

\section{MENSAJES CLAVE}

Motivación para realizar el estudio: La infección por el virus SARS-CoV-2 afecta en diferente medida a la población pediátrica, aunque la gran mayoría desarrolla enfermedad leve, algunos requieren hospitalizarse e incluso ingresar a cuidados intensivos.

Principales hallazgos: Se hospitalizaron 125 pacientes, $16,8 \%$ presentó síndrome inflamatorio multisistémico, mientras que $18,4 \%$ ingresó a la unidad de cuidados intensivos pediátricos. El 4,8\% (n=6) falleció, 3 fueron del grupo en estado crítico y ninguno del grupo con SIM.

Implicancias: Describir las características de los niños infectados con SARS-CoV-2 con mayores necesidades de asistencia médica ayudaría a mejorar la planificación del uso de los servicios de salud en esta población.

y una combinación entre rash, inyección conjuntival, manifestaciones gastrointestinales, coagulopatía, hipotensión y signos de disfunción miocárdica o inflamación mucocutánea, según los lineamientos de la OMS ${ }^{(13)}$. Además, se consideraron pacientes en estado crítico a los que ingresaron a la UCIP por dificultad respiratoria, signos de shock, encefalopatía, falla cardiaca o necesidad de monitoreo continuo.

En todos los casos se recolectó la información del primer registro en la historia clínica durante la hospitalización en sala general o en la UCIP.

Las variables evaluadas en todos los pacientes fueron las características epidemiológicas y clínicas, como sexo, edad en años cumplidos, antecedente de contacto con persona con COVID-19 y se diferenció el lugar de procedencia en zona céntrica (si procedían del departamento de Lima o la provincia constitucional del Callao) y periferia para el resto del país. También se consideraron los antecedentes de comorbilidades (presencia del antecedente de cualquier tipo de enfermedad crónica), tiempo de enfermedad por COVID-19, taquipnea (frecuencia respiratoria mayor a 50 por minuto para menores de 1 año, mayor a 40 entre 1 y 5 años, y superior a 28 para mayores de 5 años), taquicardia (frecuencia cardiaca mayor a 160 por minuto para menores de 2 años y más de 140 de 2 años a más), desaturación (saturación de oxígeno menor a 92\%) y otros signos y síntomas predominantes, el resultado positivo de la prueba molecular o de anticuerpos IgM o IgG, y la ocurrencia de fallecimiento.

Las variables laboratoriales fueron el recuento del número de leucocitos $\left(\mathrm{x} 10^{3} / \mu \mathrm{L}\right)$, linfocitos $\left(\mathrm{x} 10^{3} / \mu \mathrm{L}\right)$, neutrófilos $\left(\mathrm{x} 10^{3} / \mu \mathrm{L}\right)$ y plaquetas $\left(\mathrm{x} 10^{3} / \mu \mathrm{L}\right)$, valor de PCR en $\mathrm{mg} / \mathrm{dL}$, deshidrogenasa láctica (DHL) en $\mathrm{U} / \mathrm{L}$, ferritina $(\mathrm{ng} / \mathrm{mL})$, dímero $D(\mu \mathrm{g} / \mathrm{mL})$, creatina fosfocinasa $(\mathrm{CPK})$ en $\mathrm{U} / \mathrm{L}, \mathrm{CPK}$ fracción $\mathrm{MB}(\mathrm{U} / \mathrm{L})$, aspartato aminotransferasa (AST) en $\mathrm{U} / \mathrm{L}$, alanina aminotransferasa (ALT) en U/L y creatinina $(\mathrm{mg} / \mathrm{dL})$. 
En el grupo en estado crítico se valoró además los días en UCIP, el uso y número de días en ventilación mecánica, el uso de cánula nasal de alto flujo (CNAF) y el número de catecolaminas y antibióticos utilizados.

\section{Análisis estadístico}

Los datos se extrajeron a una hoja de cálculo en el programa Microsoft Excel ${ }^{\circledR}$ versión16.42 a partir de los registros clínicos digitales que maneja la institución. Este procedimiento fue realizado de manera independiente por dos miembros del equipo de investigación (CGR y PBS) usando la técnica de la doble digitación. El análisis estadístico se realizó en el programa Stata versión 15.0 (StataCorp, TX, EUA). Para los análisis descriptivos se usaron frecuencias relativas y absolutas en las variables cualitativas y medidas de tendencia central y dispersión en las cuantitativas según normalidad o no. Se realizaron análisis estratificados de acuerdo con el estado crítico y el diagnóstico de SIM.

\section{Consideraciones éticas}

El presente estudio fue evaluado y aprobado por el comité de ética institucional del Hospital Nacional Edgardo Rebagliati Martins. Al ser un estudio retrospectivo no se utilizó consentimiento informado.

\section{HALLAZGOS}

La muestra incluyó 125 pacientes pediátricos hospitalizados por cualquier causa e infección por SARS-CoV-2. La edad media fue 6,5 años (DE: 4,4); el 58,4\% $(\mathrm{n}=73)$ fueron varones; el $42,4 \%(n=53)$ tenía alguna comorbilidad; la mediana de tiempo de enfermedad fue de 3 días (RIC: 2-7). El 16,8\% $(\mathrm{n}=21)$ de los pacientes presentaron criterios de SIM; mientras que el $18,4 \%(\mathrm{n}=23)$ ingresaron a la UCIP. El 60,9\% $(n=14)$ de los pacientes en estado crítico y el $85,7 \%(n=18)$ del grupo con SIM fueron previamente sanos. Además, el $100 \%(\mathrm{n}=21)$ del grupo con SIM tuvieron anticuerpos IgG (Tabla 1).

Tabla 1. Características clínicas y epidemiológicas de niños con infección por SARS-CoV-2 de acuerdo con el estado crítico y la presencia del síndrome inflamatorio multisistémico $(\mathrm{n}=125)$ en un hospital de referencia en Lima, Perú.

\begin{tabular}{|c|c|c|c|c|c|}
\hline \multirow{3}{*}{ Variable } & \multirow{2}{*}{$\begin{array}{l}\text { Total } \\
\mathbf{n}(\%) \\
\end{array}$} & \multicolumn{2}{|c|}{ Estado crítico } & \multicolumn{2}{|c|}{ Síndrome inflamatorio multisistémico } \\
\hline & & Presente & Ausente & Presente & Ausente \\
\hline & & n (\%) & n (\%) & n (\%) & n (\%) \\
\hline Total & $125(100)$ & $23(18,4)$ & $102(81,6)$ & $21(16,8)$ & $104(83,2)$ \\
\hline Edad en años & $6,5(4,4)^{*}$ & $7,4(3,8)^{*}$ & $6,4(4,5)^{*}$ & $6(3-8)^{* *}$ & $7(3-11)^{* *}$ \\
\hline \multicolumn{6}{|l|}{ Sexo } \\
\hline Mujer & $52(41,6)$ & $10(43,5)$ & $42(41,2)$ & $10(47,6)$ & $42(40,4)$ \\
\hline Varón & $73(58,4)$ & $13(56,5)$ & $60(58,8)$ & $11(52,4)$ & $62(59,6)$ \\
\hline \multicolumn{6}{|l|}{ Lugar de procedencia ${ }^{\ddagger}$} \\
\hline Zona céntrica & $94(75,2)$ & $14(60,8)$ & $80(78,4)$ & $16(76,2)$ & $78(75,0)$ \\
\hline Periferia & $31(24,8)$ & $9(39,2)$ & $22(21,6)$ & $5(23,8)$ & $26(25,0)$ \\
\hline \multicolumn{6}{|l|}{ Comorbilidad } \\
\hline Sí & $53(42,4)$ & $9(39,1)$ & $44(43,1)$ & $3(14,3)$ & $50(48,1)$ \\
\hline No & $72(57,6)$ & $14(60,9)$ & $58(56,9)$ & $18(85,7)$ & $54(51,9)$ \\
\hline \multicolumn{6}{|l|}{ Antecedente de contacto } \\
\hline Sí & $57(46,3)$ & $14(60,9)$ & $43(43,0)$ & $15(71,4)$ & $42(41,2)$ \\
\hline No & $66(53,7)$ & $9(39,1)$ & $57(57,0)$ & $6(28,6)$ & $60(58,8)$ \\
\hline Tiempo de enfermedad(días) & $3(2-7)^{* *}$ & $5(3-7)^{* *}$ & $3(1-7)^{\star *}$ & $4(3-5)^{\star *}$ & $3(1-7)^{\star *}$ \\
\hline \multicolumn{6}{|l|}{ Prueba molecular } \\
\hline Positiva & $15(22,8)$ & $5(22,7)$ & $10(22,7)$ & $1(8,3)$ & $14(25,9)$ \\
\hline Negativa & $51(77,2)$ & $17(77,3)$ & $34(77,3)$ & $11(91,7)$ & $40(74,1)$ \\
\hline \multicolumn{6}{|l|}{ Anticuerpos IgM } \\
\hline Presentes & $66(52,8)$ & $8(34,8)$ & $58(56,9)$ & $6(28,6)$ & $60(57,7)$ \\
\hline Ausentes & $59(47,2)$ & $15(65,2)$ & $44(43,1)$ & $15(71,4)$ & $44(42,3)$ \\
\hline \multicolumn{6}{|l|}{ Anticuerpos IgG } \\
\hline Presentes & $108(86,4)$ & $18(78,3)$ & $90(88,2)$ & $21(100)$ & $87(83,6)$ \\
\hline Ausentes & $17(13,6)$ & $5(21,7)$ & $12(11,8)$ & $0(0)$ & $17(16,4)$ \\
\hline
\end{tabular}

${ }^{*}$ Media (desviación estándar), ${ }^{* *}$ mediana (rango intercuartílico), $\ddagger$ se consideró como zona céntrica al departamento de Lima y la provincia del Callao y como periferia al resto del país. 
La mediana del recuento de plaquetas fue $173000 / \mu \mathrm{L}$ en el grupo en estado crítico y $152000 / \mu \mathrm{L}$ en el grupo con SIM, mientras que fue $260000 / \mu \mathrm{L}$ y $250000 / \mu \mathrm{L}$ en los grupos que no presentaron estas condiciones (Tabla 2).

El $65,2 \%(n=15)$ del grupo en estado crítico y $95,2 \%(n=20)$ del grupo con SIM tuvo fiebre. Mientras que el 47,8\% ( $n=11)$ de los pacientes en estado crítico tuvieron disnea y ninguno del grupo con SIM presentó desaturación, ni tos (Tabla 3).

De los 23 pacientes hospitalizados en la UCIP, 14 (60,9\%) ingresaron a ventilación mecánica invasiva y $3(13 \%)$ necesitaron CNAF; la mediana de permanencia en la UCIP fue de 6 días y de 2 días para el uso de ventilación mecánica. Además, el $91,3 \%(\mathrm{n}=21)$ recibió antibióticos y el $47,8 \%(\mathrm{n}=11)$ alguna catecolamina, como epinefrina, norepinefrina o dobutamina.

Fallecieron 6 de los 125 niños (4,8\%), solo uno de ellos no presentaba comorbilidad. Tres de los 6 fallecidos fueron del grupo en estado crítico (13,0\%). Los otros tres, aunque estaban graves, no formaron parte de este grupo debido a que no lograron ingresar a la UCIP por falta de camas disponibles. El primero, de los que falleció fuera de la UCIP fue un paciente con síndrome de intestino corto con sepsis de foco abdominal. El segundo, un paciente con neuroblastoma, y el tercero, un paciente con falla hepática, todos fallecieron con neumonía por SARS-CoV-2. Ninguno de los 21 pacientes con SIM falleció.

\section{DISCUSIÓN}

De los 125 niños y adolescentes hospitalizados con infección por SARS-CoV-2, el 18,4\% ingresó a la UCIP, porcentaje similar a lo reportado en hospitales de Inglaterra $(18 \%)^{(14)}$, aunque, otras series reportan valores más extremos (del 6 al $33,2 \%)^{(15,16)}$. Los criterios usados para determinar el ingreso a la UCIP, además de la disponibilidad de camas en diferentes fases de la pandemia, podrían explicar esta diferencia. El SIM se presentó en $16,8 \%$, porcentaje mayor al $10,8 \%$ reportado por Kim LK et al. ${ }^{(16)}$ durante un mes de recolección de datos y, aunque ambos estudios usaron los mismos criterios diagnósticos, el tiempo de recolección en nuestro estudio fue mayor.

La proporción de varones y mujeres afectados fue similar para los pacientes críticos como para los que desarrollaron SIM, otros autores también encontraron lo mismo ${ }^{(17)}$. Coincidimos con publicaciones previas ${ }^{(16-18)}$ en que los más afectados son los niños en edad escolar. El contacto epidemiológico fue más frecuente en pacientes con SIM que en pacientes sin este cuadro; además, todos nuestros pacientes con SIM presentaron anticuerpos IgG contra el SARSCoV-2, en otras series hasta el 87,0\% de pacientes fueron reactivos a este anticuerpo ${ }^{(7)}$. Debido a que la presentación clínica inicial es inespecífica, tanto el antecedente de contacto como la positividad de la IgG podrían permitir la sospecha diagnóstica.

El 42,4\% de todos los niños evaluados presentaron alguna comorbilidad. Sin embargo, el 85,7\% de pacientes con SIM habían estado sanos, porcentaje similar a otros reportes hospitalarios ${ }^{(7,16)}$. De igual manera, el $60,9 \%$ de los que desarrollaron enfermedad crítica no tenían comorbilidades; tampoco se ha reportado relación en otros estudios ${ }^{(14)}$. No obstante, Bellino S. et al., describieron que la presencia de al menos una comorbilidad ${ }^{(5)}$ se asocia a casos graves. Estas diferencias podrían explicarse porque nuestro estudio se realizó en un centro pediátrico de referencia nacional y por lo tanto es previsible la mayor diversidad de los pacientes.

Tabla 2. Características laboratoriales de niños con infección por SARS-CoV-2, según el estado crítico y la presencia de SIM en un hospital de referencia en Lima, Perú.

\begin{tabular}{|c|c|c|c|c|c|}
\hline \multirow{2}{*}{ Variable } & \multirow{2}{*}{ Total $^{*}$} & \multicolumn{2}{|c|}{ Estado crítico } & \multicolumn{2}{|c|}{ Sindrome inflamatorio multisistémico } \\
\hline & & Presente $^{\star}$ & Ausente $^{\star}$ & Presente ${ }^{\star}$ & Ausente $^{*}$ \\
\hline Leucocitos $\left(\mathrm{x} 10^{3}\right) / \mu \mathrm{L}$ & $10,4(6,6-15,2)$ & $12,2(7,9-18,8)$ & $10,2(6,4-14,6)$ & $11,9(7,6-15,1)$ & $9,9(6,4-15,2)$ \\
\hline Linfocitos $\left(\mathrm{x} 10^{3}\right) / \mu \mathrm{L}$ & $1,8(1,0-3,0)$ & $0,7(0,4-1,9)$ & $2,0(1,3-3,1)$ & $1,4(0,4-1,9)$ & $1,9(1,2-3,0)$ \\
\hline Neutrófilos $\left(\mathrm{x} 10^{3}\right) / \mu \mathrm{L}$ & $6,7(3,5-11,1)$ & $8,9(4,0-13,1)$ & $6,5(3,3-10,2)$ & $7,2(5,5-10,9)$ & $6,6(3,3-11,2)$ \\
\hline Plaquetas $\left(\times 10^{3}\right) / \mu \mathrm{L}$ & $234(135-331)$ & $173(62-230)$ & $260(152-339)$ & $152(72-251)$ & $250(167-336)$ \\
\hline $\operatorname{PCR}(\mathrm{mg} / \mathrm{dL})$ & $4,9(1,1-17,9)$ & $17,9(2,2-25,8)$ & $4,1(1,0-13,9)$ & $22,1(15,8-25,4)$ & $2,9(0,5-11,6)$ \\
\hline DHL (U/L) & $253(201-441)$ & $245(197-516)$ & $257(201-441)$ & $245(211-403)$ & $257(195-446)$ \\
\hline Ferritina $(\mathrm{ng} / \mathrm{mL})$ & $273(111-578)$ & $575(192-1179)$ & $202(85-365)$ & $491(306-653)$ & $164,5(76,6-554)$ \\
\hline Dímero D $(\mu \mathrm{g} / \mathrm{mL})$ & $2,4(0,9-8,3)$ & $4,3(3,4-7,7)$ & $1,4(0,4-3,2)$ & $3,8(1,5-4,6)$ & $1,5(0,5-4,3)$ \\
\hline $\mathrm{CPK}$ total $(\mathrm{U} / \mathrm{L})$ & $144(65-359)$ & $105(48-359)$ & $147(117-393)$ & $117(65-359)$ & $147,5(61-393)$ \\
\hline CPK-MB (U/L) & $1,5(0,8-4,8)$ & $2,3(0,7-13,5)$ & $1,4(1,0-1,5)$ & $1,8(0,7-5,7)$ & $1,5(0,9-4,2)$ \\
\hline $\operatorname{AST}(\mathrm{U} / \mathrm{L})$ & $33(24,5-58,0)$ & $40(28-67)$ & $31(23-49)$ & $39,5(26,5-68,0)$ & $33(23-50)$ \\
\hline $\operatorname{ALT}(\mathrm{U} / \mathrm{L})$ & $25(13-45)$ & $31(17-56)$ & $21(13-42)$ & $33,5(20,5-54,0)$ & $20(13-40)$ \\
\hline Creatinina $(\mathrm{mg} / \mathrm{dL})$ & $0,4(0,3-0,6)$ & $0,4(0,4-0,6)$ & $0,4(0,3-0,5)$ & $0,5(0,4-0,6)$ & $0,4(0,3-0,5)$ \\
\hline
\end{tabular}

* Mediana (rango intercuartílico); PCR: proteína C reactiva; DHL: deshidrogenasa láctica; CPK: creatina fosfocinasa; AST: aspartato aminotransferasa; ALT: alanina aminotransferasa. 
Tabla 3. Signos y síntomas en niños con infección por SARS-CoV-2, en estado crítico con sindrome inflamatorio multisistémico internados en un hospital de referencia en Lima, Perú $(\mathrm{n}=125)$.

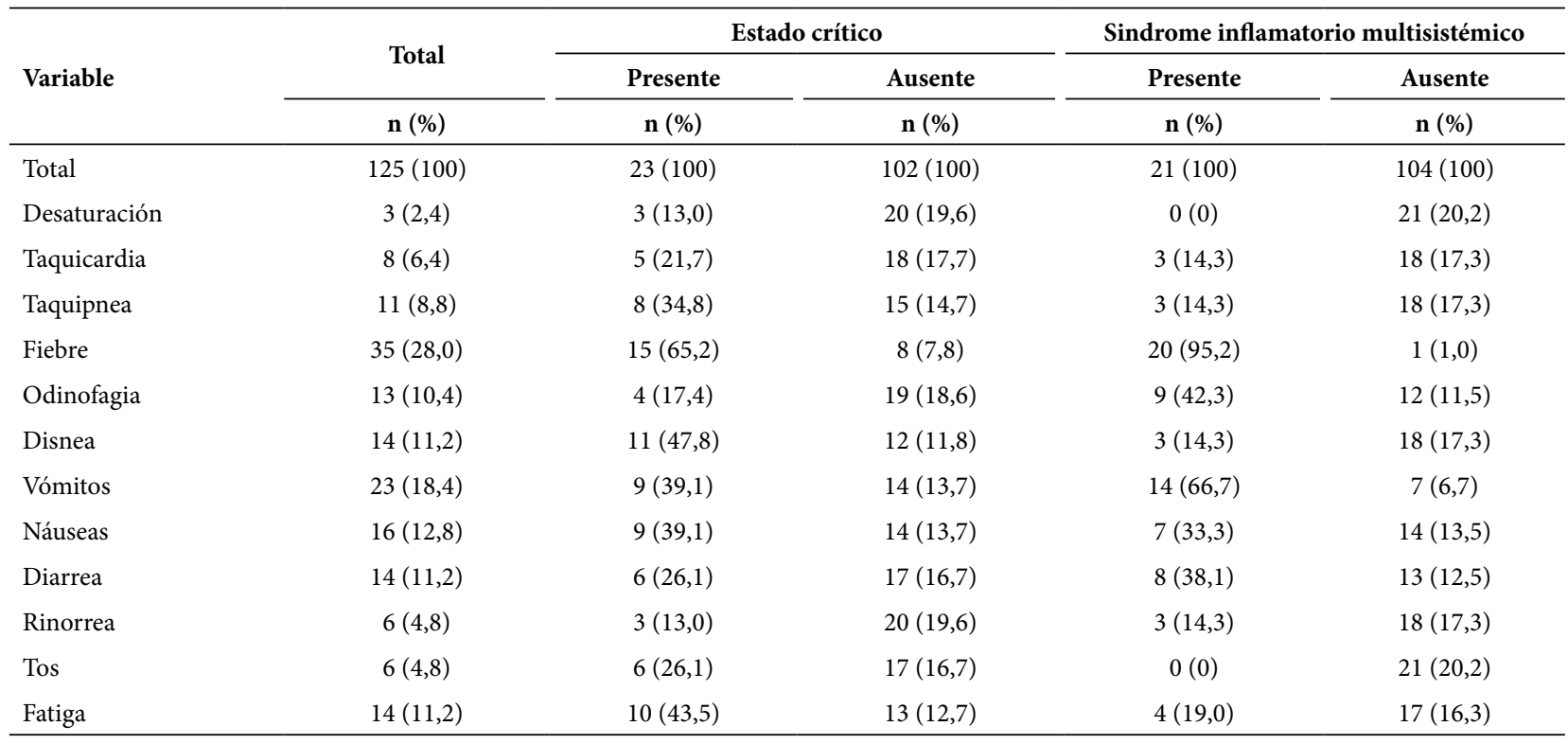

Las manifestaciones clínicas más frecuentes del grupo en estado crítico fueron fiebre $(65,2 \%)$ y disnea $(47,8 \%)$. Similar a lo reportado por Shekerdemian et al. ${ }^{\left({ }^{(1)}\right)}$ y Derespina et al. ${ }^{(20)}$ que muestran mayor compromiso respiratorio en los niños en estado crítico con infección por SARS-CoV-2. En los que desarrollaron SIM, la fiebre $(95,2 \%)$ y los vómitos $(66,7 \%)$ fueron las más prevalentes. Coincidimos con otros autores en que las manifestaciones gastrointestinales son frecuentes ${ }^{(7-9)}$; en este grupo ninguno presentó tos, ni desaturación. A nivel laboratorial el menor recuento de linfocitos y plaquetas, y los mayores valores de PCR, ferritina y dímero D reflejan el estado inflamatorio que caracteriza a ambas enfermedades. ${ }^{(7-9,20)}$

Los 23 pacientes ingresados a la UCIP recibieron tratamiento de soporte de acuerdo con sus necesidades. El 60,9\% fue tratado con ventilación mecánica invasiva y el $47,8 \%$ recibió alguna catecolamina. El porcentaje de ventilación invasiva fue mayor y el manejo con catecolaminas menor que lo reportado en niños del Reino Unido ${ }^{(8)}$. Además, el 13\% solo necesitó CNAF, resultado similar al 20\% que reportó Derespina et al. en 70 niños con infección por SARS-CoV-2 tratados en la UCIP ${ }^{(20)}$.

Seis $(4,8 \%)$ de los 125 pacientes con infección por SARS-CoV-2 fallecieron, porcentaje similar al 5,2\% reportado por Bhumbra et al. en 19 niños internados hasta mayo del 2020 en el Hospital de la Universidad de Indiana en Estados Unidos ${ }^{(4)}$. Tres (13\%) de los fallecidos fueron del grupo en estado crítico; el resto falleció en sala de cuidados generales en relación con otras comorbilidades. Ninguno de los pacientes con SIM falleció; al igual que en reportes previos ${ }^{(9,14)}$.

Nuestro estudio tiene limitaciones. Se realizó en un solo hospital y, aunque se trata del centro más importante de referen- cia nacional, la muestra no es representativa de la población general. Por ser un estudio retrospectivo, el sesgo de información está presente. $\mathrm{Al}$ incluir pacientes hospitalizados por cualquier causa es probable que algunos pacientes tuvieran un motivo de hospitalización diferente a la infección por SARS-CoV-2 y esta sea una infección asintomática. Lo ideal hubiera sido excluirlos, pero esta distinción escapa del objetivo del estudio. No incluimos exámenes de imágenes, por tanto, la afectación pulmonar y cardiaca no fue evaluada, aunque probablemente no todos los pacientes necesitaban estos estudios.

Concluimos que en los pacientes pediátricos hospitalizados con infección por SARS-Cov-2, los pacientes en estado crítico se presentaron en un $18,4 \%$ y con SIM en $16,8 \%$ con una proporción similar en varones y mujeres. La mortalidad general fue de $4,8 \%$ y de $13,0 \%$ en los que ingresaron a la UCIP. Aún existen áreas de incertidumbre, como la evolución a largo plazo; sin embargo, creemos que los datos encontrados servirán de base para plantear hipótesis en futuras investigaciones y dirigir el criterio de los tomadores de decisiones.

Agradecimientos: Al Dr. Carlos French Yrigoyen, gerente clínico del HNERM, por las gestiones administrativas que permitieron desarrollar la investigación.

Contribuciones de autoría: RRP, PLQ, CGR, LCY, ECP, PBS e IPT participaron en la concepción, redacción y revisión crítica del artículo. Además, CGR y RRP realizaron los trámites para la aprobación por el comité de ética. PBS y CGR se encargaron de la recolección de datos. IPT, PLQ y RRP realizaron el análisis de los datos, interpretación y discusión de estos. Todos los autores aprobaron la versión final del artículo.

Financiamiento: La investigación fue autofinanciada.

Conflictos de interés: Ninguno de los autores tiene algún conflicto de interés que declarar. 


\section{REFERENCIAS BIBLIOGRÁFICAS}

1. World Health Organization. WHO. 2020 [citado 26 de octubre de 2020]. Disponible en: https://www.who.int/director-general/speeches/ detail/who-director-general-s-opening-remarks-at-the-media-briefing-on-covid-19---11-march-2020

2. COVID-19 Map [Internet]. Johns Hopkins Coronavirus Resource Center. [citado 26 de octubre de 2020]. Disponible en: https://coronavirus.jhu.edu/map.html

3. Ludvigsson JF. Systematic review of COVID-19 in children shows milder cases and a better prognosis than adults. Acta Paediatr. 2020;109(6):1088-95. doi: 10.1111/apa.15270.

4. Bhumbra S, Malin S, Kirkpatrick L, Khaitan A, John CC, Rowan CM, et al. Clinical Features of Critical Coronavirus Disease 2019 in Children. Pediatr Crit Care Med. 2020;21(10):948-53. doi: 10.1097/ PCC. 0000000000002511.

5. Bellino S, Punzo O, Rota MC, Del Manso M, Urdiales AM, Andrianou X, et al. COVID-19 Disease Severity Risk Factors for Pediatric Patients in Italy. Pediatrics. 2020;146(4):e2020009399. doi: 10.1542/ peds.2020-009399.

6. Guo C-X, He L, Yin J-Y, Meng X-G, Tan W, Yang G-P, et al. Epidemiological and clinical features of pediatric COVID-19. BMC Med. 2020;18(1):250. doi: 10.1186/s12916-020-01719-2.

7. Whittaker E, Bamford A, Kenny J, Kaforou M, Jones CE, Shah P, et al. Clinical Characteristics of 58 Children With a Pediatric Inflammatory Multisystem Syndrome Temporally Associated With SARS-CoV-2. JAMA. 2020;324(3):259. doi: 10.1001/jama.2020.10369.

8. Davies P, Evans C, Kanthimathinathan HK, Lillie J, Brierley J, Waters G, et al. Intensive care admissions of children with paediatric inflammatory multisystem syndrome temporally associated with SARS-CoV-2 (PIMSTS) in the UK: a multicentre observational study. Lancet Child Adolesc Health. 2020;4(9):669-77. doi: 10.1016/S2352-4642(20)30215-7.

9. Toubiana J, Poirault C, Corsia A, Bajolle F, Fourgeaud J, Angoulvant F, et al. Kawasaki-like multisystem inflammatory syndrome in children during the covid-19 pandemic in Paris, France: prospective observational study. BMJ. 2020;m2094. doi: 10.1136/bmj.m2094.

10. Pouletty M, Borocco C, Ouldali N, Caseris M, Basmaci R, Lachaume $\mathrm{N}$, et al. Paediatric multisystem inflammatory syndrome temporally associated with SARS-CoV-2 mimicking Kawasaki disease (Kawa-COVID-19): a multicentre cohort. Ann Rheum Dis. 2020;79(8):999-1006. doi: 10.1136/annrheumdis-2020-217960.

11. EsSalud W. Hospital Rebagliati de EsSalud alcanza máxima categoría por su alta especialidad y capacidad resolutiva. EsSalud [Internet]. [citado 3 de noviembre de 2020]. Disponible en: http://www.essalud. gob.pe/hospital-rebagliati-de-essalud-alcanza-maxima-categoria-por-su-alta-especialidad-y-capacidad-resolutiva/.

12. Alerta epidemiológica ante riesgo de intensificación de la transmisión comunitaria de COVID-19 en el periodo post cuarentena, en el Perú. [Internet]. Ministerio de Salud - Centro Nacional de Epidemiología, Prevención y Control de Enfermedades; 2020. Disponible en: https:// www.dge.gob.pe/portal/docs/alertas/2020/AE019.pdf.

13. World Health Organization. Multisystem inflammatory syndrome in children and adolescents temporally related to COVID-19 [Internet]. World Health Organization. [citado 3 de noviembre de 2020]. Disponible en: https://www.who.int/news-room/commentaries/ detail/multisystem-inflammatory-syndrome-in-children-and-adolescents-with-covid-19.

14. Swann OV, Holden KA, Turtle L, Pollock L, Fairfield CJ, Drake TM, et al. Clinical characteristics of children and young people admitted to hospital with covid-19 in United Kingdom: prospective multicentre observational cohort study. BMJ. 2020;m3249. doi: 10.1136/bmj.m3249.

15. Song X, Delaney M, Shah RK, Campos JM, Wessel DL, DeBiasi RL. Comparison of Clinical Features of COVID-19 vs Seasonal Influenza A and B in US Children. JAMA Netw Open. 2020;3(9):e2020495. doi: 10.1001/jamanetworkopen.2020.20495.

16. Kim L, Whitaker M, O'Halloran A, Kambhampati A, Chai SJ, Reingold A, et al. Hospitalization Rates and Characteristics of Children Aged $<18$ Years Hospitalized with Laboratory-Confirmed COVID-19COVID-NET, 14 States, March 1-July 25, 2020. MMWR Morb Mortal Wkly Rep. 2020;69(32):1081-8. doi: 10.15585/mmwr.mm6932e3.

17. Ding Y, Yan H, Guo W. Clinical Characteristics of Children With COVID-19: A Meta-Analysis. Front Pediatr. 2020;8:431. doi: 10.3389/ fped.2020.00431.

18. Meena J, Yadav J, Saini L, Yadav A, Kumar J. Clinical Features and Outcome of SARS-CoV-2 Infection in Children: A Systematic Review and Meta-analysis. Indian Pediatr. 2020;57(9):820-6. doi: 10.1007/ s13312-020-1961-0.

19. Shekerdemian LS, Mahmood NR, Wolfe KK, Riggs BJ, Ross CE, McKiernan CA, et al. Characteristics and Outcomes of Children With Coronavirus Disease 2019 (COVID-19) Infection Admitted to US and Canadian Pediatric Intensive Care Units. JAMA Pediatr. 2020;174(9):868. doi: 10.1001/jamapediatrics.2020.1948.

20. Derespina KR, Kaushik S, Plichta A, Conway EE, Bercow A, Choi J, et al. Clinical Manifestations and Outcomes of Critically Ill Children and Adolescents with Coronavirus Disease 2019 in New York City. J Pediatr. 2020;226:55-63.e2. doi: 10.1016/j.jpeds.2020.07.039. 